\title{
Diagnostic and interventional magnetic resonance neurography diagnosis of brachytherapy seed-mediated pudendal nerve injury: a case report
}

\author{
Luke W. Bonham ${ }^{1}$, Amin S. Herati ${ }^{2}$, Edward F. McCarthy ${ }^{3}$, A. Lee Dellon ${ }^{4}$, Jan Fritz ${ }^{1,5}$ \\ ${ }^{1}$ Russell H. Morgan Department of Radiology and Radiological Science, ${ }^{2}$ Department of Urology, ${ }^{3}$ Department of Pathology, ${ }^{4}$ Departments of \\ Plastic Surgery and Neurosurgery, Johns Hopkins University School of Medicine, Baltimore, MD, USA; ${ }^{5}$ Department of Radiology, New York \\ University School of Medicine and Langone Heath, New York, NY, USA \\ Correspondence to: Jan Fritz, MD. Department of Radiology, New York University School of Medicine and Langone Heath, 660 1st Ave, 3rd Floor, \\ Rm 313, New York, NY 10016, USA. Email: jan.fritz@nyulangone.org.
}

\begin{abstract}
Injury to the pudendal nerve in men presents with pain, paresthesia, or numbness of the perineum, and/or scrotum, and/or penis. There is evidence implicating the brachytherapy seeds used to treat prostate cancer as source of pudendal nerve injury. Compared to surgical prostatectomy, brachytherapy has the advantage of being less invasive, but seeds may not only lead to well-established complications such as urinary, bowel, and erectile dysfunction, but also injury to the sensory branches of the pudendal nerve. We report and document a case of pudendal nerve injury secondary to brachytherapy seeds diagnosed with magnetic resonance (MR) neurography, nerve blocks, and histopathological examination; and successful treatment via sensory branch neurectomy.
\end{abstract}

Keywords: Pudendal nerve; magnetic resonance imaging (MRI); brachytherapy; peripheral nerve injuries; prostatic neoplasms; case report

Submitted Dec 24, 2019. Accepted for publication Mar 04, 2020.

doi: $10.21037 /$ tau.2020.03.22

View this article at: http://dx.doi.org/10.21037/tau.2020.03.22

\section{Introduction}

Morbidity following prostate brachytherapy is relatively common, with approximately $50 \%$ of men reporting a complication within 2 years of the procedure (1). The mechanism of injury generally stems from the location of the radioactive brachytherapy seeds, which are placed into the prostate (2). The radioactive brachytherapy seeds ideally produce only local damage to the cancerous regions but may also irradiate neighboring structures. Urinary symptoms are most frequent (34\%), followed by bowel (21\%), and erectile $(17 \%)$ symptoms $(1,3)$. Suspected cases of pudendal nerve injury following brachytherapy are less common and often exhibit chronic pain, hyperesthesia, and paresthesia of the sensory innervations of the pudendal nerve $(4,5)$. These symptoms make common activities such as urinating, sitting, or having intercourse difficult and result in substantial reductions in quality of life. Case reports of chronic pain following brachytherapy exist, but appear complicated by other comorbidities such as chronic bacterial prostatitis (5). Further, there are no confirmed cases of pudendal nerve injury in which the brachytherapy seeds were visualized with certainty near the pudendal nerve.

Diagnosis of pudendal nerve injury is complex and requires careful evaluation of other potential sources of perineal symptoms such as injury to the posterior femoral cutaneous nerve $(6,7)$. Demonstrating the etiology of pudendal nerve injury after brachytherapy is complicated by difficulty visualizing damage to the pudendal nerve and its surrounding structures. There are reports of computed tomography visualization of brachytherapy seeds in the regions commonly traversed by the pudendal nerve (4), but the low contrast resolution of computed tomography does not permit visualization of the pudendal nerve itself.

High-resolution magnetic resonance neurography at 3 

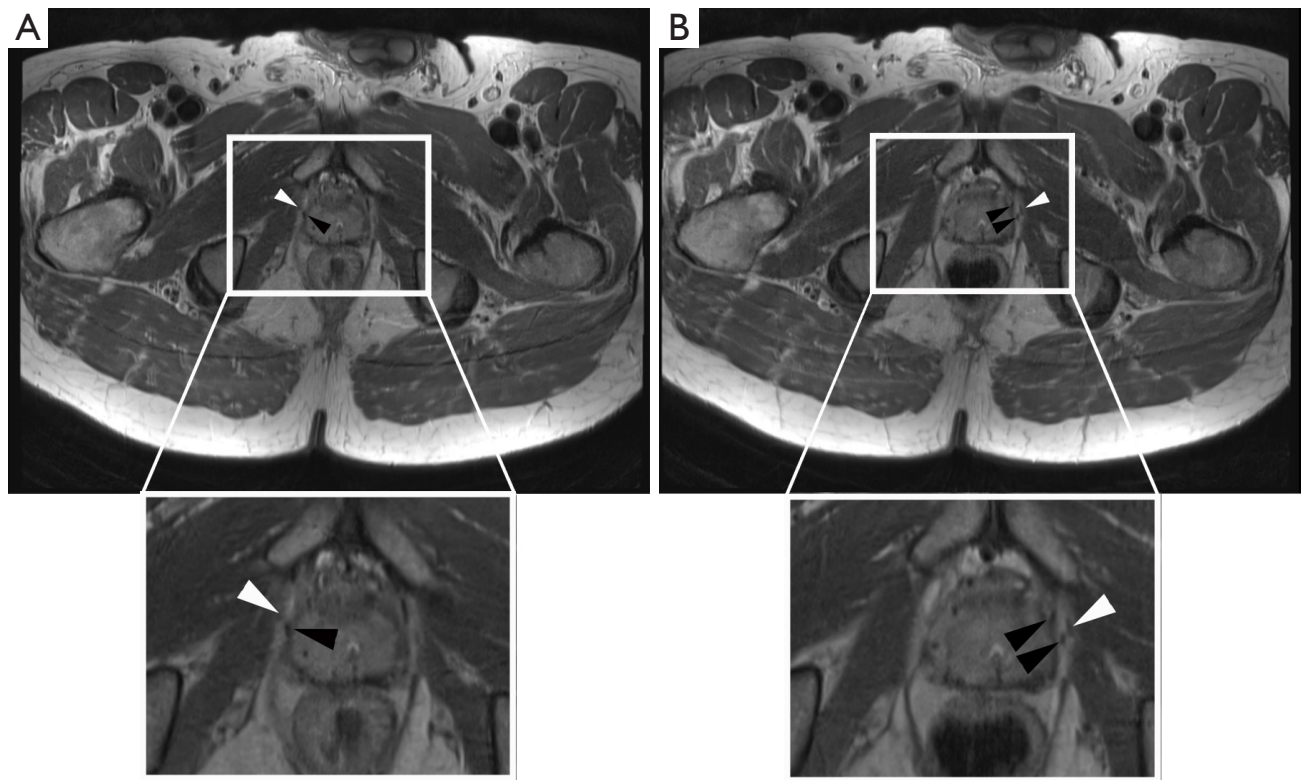

Figure 1 Diagnostic MR neurography. (A) Axial proton density-weighted magnetic resonance neurography (MRN) image illustrating a brachytherapy seed (black arrow) in close proximity to the right perineal branches (white arrow) of the pudendal nerve; (B) axial proton density-weighted MRN image illustrating brachytherapy seeds (black arrows) in close proximity to the left perineal branch (white arrow) of the pudendal nerve.

Tesla (3T MRN) enables the visualization of small nerves and their surrounding soft tissue structures, and can often diagnose peripheral nerve injury and neuropathy (8). However, 3T MRN may be limited in the setting of brachytherapymediated injury due to magnetic resonance (MR) image artifacts created by metallic brachytherapy seeds. Using highresolution metal artifact reduction MRN techniques (9), we report a case presenting with signs and symptoms of pudendal nerve injury after brachytherapy seed placement, where 3T MRN demonstrated the seeds in close proximity with the pudendal nerves, subsequent confirmation of pudendal nerve-mediated symptoms through selective nerve blocks, and successful treatment through surgical resection of the perineal branches of the pudendal nerve. We present the following case in accordance with the CARE reporting checklist (available at http://dx.doi.org/10.21037/ tau.2020.03.22).

\section{Case presentation}

The data presented in this case report were obtained under an internal review board-approved protocol for prospective data collection and with informed consent from the patient.

A 67-year-old man with a past medical history of hyperlipidemia, benign prostatic hyperplasia (BPH), and prostate cancer (Gleason score $3+4=7$ ) presented to our clinic with intractable pain of the penis and scrotum accompanied by a burning sensation during urination that was so severe he could not void completely. The symptoms developed within one month following brachytherapy treatment consisting of 104 brachytherapy seeds that were placed with transrectal ultrasonography guidance for a local dose of $125 \mathrm{~Gy}$. The patient's symptoms consisted of excruciating pain on urination with an International Prostate Symptom Score (10) of 35/35, urinary bother symptom score (10) of $6 / 6$, and a pain severity of $10 / 10$ on an 11-point visual analog scale where 10 is the maximum imaginable pain. The patient's pain was exacerbated by sitting and there were no sensory or motor deficits.

Cystoscopy showed normal urethra and bladder. Intrarectal diazepam, oral amitriptyline, pelvic floor physical therapy, tadalafil, and acupuncture were ineffective for pain control, whereas morphine treatment induced a pain reduction from $10 / 10$ to $8.5 / 10$. However, intrarectal diazepam and amitriptyline made urination easier.

High-resolution 3T MRN with high bandwidthbased metal artifact reduction technique (9) showed the brachytherapy seeds located in close proximity to the right 

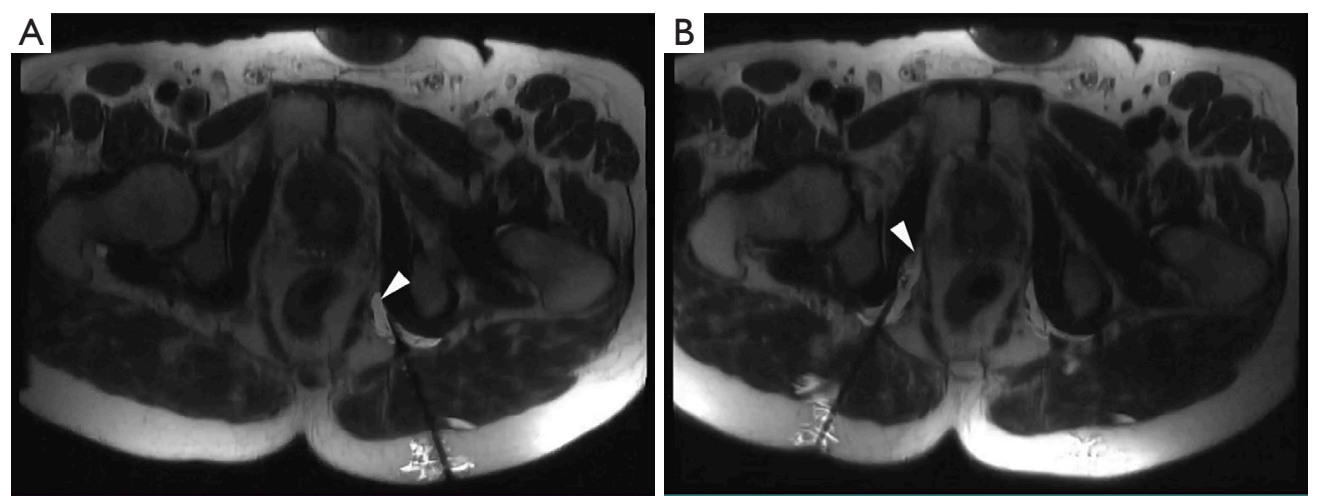

Figure 2 MR-guided selective pudendal nerve block. (A) Axial interventional magnetic resonance neurography (MRN) image at level of the prostate shows the left pudendal nerve and surrounding tissue. Anesthetic with steroids surrounds the left pudendal nerve (white arrow). (B) Axial MRN image at level of the prostate shows the right pudendal nerve and surrounding tissue. Anesthetic with steroids surrounds the right pudendal nerve (white arrow).

and left pudendal nerves (Figure 1), raising the possibility of radiation-induced pudendal nerve injury. Because the MRN appearance of the pudendal nerves was otherwise normal without findings to indicate neuropathy, selective MRNguided nerve blocks were performed in Alcock's canal and proximal to the perineal branches, in order to test the hypothesis that the pudendal nerve was the pain generator.

Prior to procedure, a urination challenge produced $5 / 10$ pain on a visual analogue scale. The procedure utilized a clinical 3T MRI system. Two 20-gauge MR-conditional injection needles were advanced under intermittent MRI using metal artifact reduction MRI into the right and left posterior aperture of Alcock's canal, just next to the pudendal nerves (11). Each pudendal canal was injected with $7 \mathrm{~mL}$ of local anesthetic mixed with long acting corticosteroid $(6 \mathrm{~mL}$ of $1 \%$ ropivacaine mixed and $1 \mathrm{~mL} / 6$ $\mathrm{mg}$ of betamethasone injectable suspension) (Figure 2). Procedural MRN images confirmed distention of the pudendal canal and circumferential perineural distribution of the injectants around the pudendal nerves on each side. There were no complications.

Sixty minutes after the procedure, the patient was able to urinate normally, which was painless and similar to his pre-brachytherapy state. In addition, sitting on a chair with a hard surface did not produce his typical sitting pain over a period of 20 minutes. On the evening of the day of the nerve block procedure, his symptoms recurred. The functional results of the perineural blocks confirmed the pudendal nerves as the pain generators and supported the hypothesis that the radioactive brachytherapy seeds located next to the pudendal nerves were inducing a local neuropathy. Over the following 2 weeks, there were no symptom-relieving effects attributable to the injected perineural steroids.

Given the patient's favorable response to pudendal nerve blocks, the patient and care team discussed the risks, benefits, and alternatives to surgical resection of the perineal branches of the right and left pudendal nerves (12). The patient elected to undergo the operation after providing written informed consent. The operation was conducted under general anesthesia and without complications by two experienced surgeons. Briefly, the patient was placed in the dorsal lithotomy position and 4-cm incisions made bilaterally using a No. 15 blade. Bipolar cautery and sharp dissection were used to visualize the ischiorectal fossae. Fibrosis in the ischiorectal fossae was noted, with shiny white scar tissue replacing native adipose tissue on the left more than right (Figure 3). The perineal branches of the pudendal nerves were identified (Figure 3) and traced back to the exit of the Alcock's canal where the perineal branches were cauterized. Of note, the patient's left perineal branch of the pudendal nerve was grossly thicker than the right branch. The resected tissue was sent for pathological analysis. The proximal branches of each nerve were then buried in Alcock's canal. Bleeding during the operation was minimal and all incisions were closed in standard fashion.

Two weeks after the operation, the patient reported significant improvement in his pain and quality of life as well as remained sexually active. Of note, he no longer required morphine, amitriptyline, or intrarectal diazepam to 

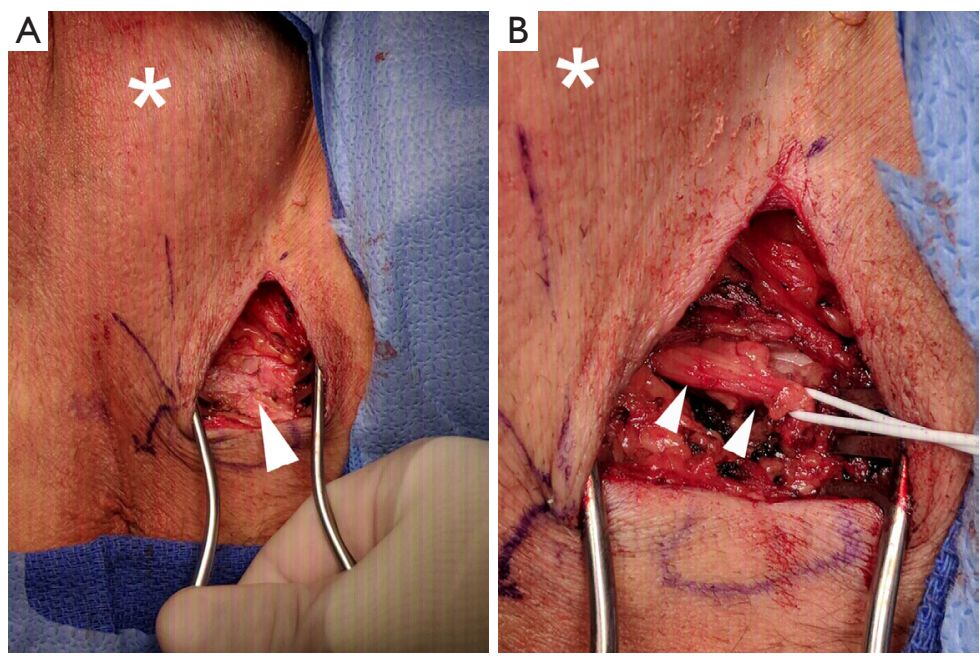

Figure 3 Intraoperative images of perineal branches of pudendal nerve during resection. (A) Intraoperative photograph of the left-sided incision into the ischial rectal fossa demonstrates shiny white scar tissue secondary to radiation where there should be just yellow fat (white arrow). The patient's scrotum is on located on the upper left side of the image (asterisk). (B) Intraoperative photograph demonstrating the left perineal branches of pudendal nerve (in white vessel loop) prior to resection (white arrows). The patient's scrotum is on located on the upper left side of the image (asterisk).

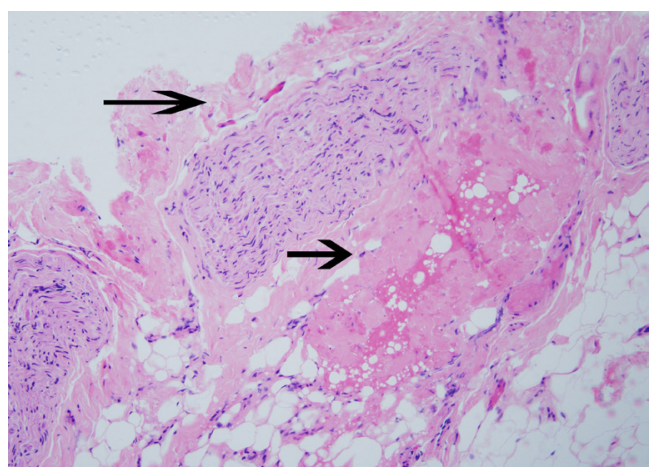

Figure 4 Photomicrograph of resected perineal branch of pudendal nerve. Microscopic photomicrograph of hematoxylin and eosin stained tissue samples at $200 \times$ magnification demonstrates areas of scattered perineural fibrosis (black arrows) around the resected nerve tissue, which is suggestive of inflammation-induced scarring secondary to brachytherapy seeds.

urinate. His exam was stable at 3-, 7-, 16-month follow-up visits. Figure $S 1$ provides a detailed timeline of the patient's diagnostic and therapeutic course.

Histopathological examination of the resected nerve branches showed peripheral nerve tissue with areas of perineural fibrosis and otherwise healthy nerve tissue (Figure 4).

\section{Discussion}

We report the successful diagnosis and treatment of pudendal nerve injury secondary to prostate brachytherapy seeds with diagnostic and interventional 3T MRN and subsequent surgical decompression and sensory branch neurectomy, which resulted in resolution of patient's pain.

Diagnosis of pain secondary to pudendal nerve injury should be confirmed by a carefully performed nerve block $(6,13)$. In our case report, we illustrate the utility of 3T MRN for detailed visualization of the causative brachytherapy seeds driving our patient's pain and utility of 3T MRN-guided injections to provide functional proof of nerve injury. Previous reports hypothesized that brachytherapy-mediated pain may be relieved by a pudendal nerve block $(4,5)$ and that brachytherapy seeds can be visualized in the regions traversed by the pudendal nerve (4). Our report provides first the proof of this theory by illustrating both successful nerve block and visualization of brachytherapy seeds in close proximity to the pudendal nerve following brachytherapy, and second confirmation by successful surgical treatment. Using photomicrographs from the patient's surgical pathology samples we also illustrate perineural fibrosis-a sign suggestive of inflammation and subsequent perineural scarring secondary to localized radiation exposure from the brachytherapy 
seeds. Thus, we document that brachytherapy seeds can cause pudendal nerve injury and should be considered in the differential diagnosis of pudendal nerve injury. Beyond this, the close correlation between the proximity of brachytherapy seeds to the pudendal nerve on 3T MRN suggests that high-resolution MRN is a useful tool during the diagnostic work-up, especially when a patient presents with mixed urinary symptoms such as pain with voiding along with urinary hesitancy with stream weakness. As was the case in this patient, two conditions may be present and successful identification of nerve injury in addition to $\mathrm{BPH}$ altered surgical planning and clinical management. While the post-surgical resolution of this patient's pain during urination provides strong evidence of successful treatment of an existing pudendal nerve injury, his multiple urinary symptoms may have had confounding effects on the pain responses. As this study describes the diagnosis and treatment of pudendal nerve injury by radioactive prostate seeds in a single patient, the conclusions may not be readily generalizable to the general population, but require confirmatory outcome data from a well-designed prospective study.

Treatment for pudendal nerve injury is multimodal and includes both oral pain medications, perineural steroid injections, thermal ablation including cryoneurolysis and radiofrequency ablation, and surgical resection or decompression (14-17). In our case, local anesthetics with steroid injections provided short-term pain relief allowing for a diagnosis of pudendal nerve-mediate pain, but the steroids did not result in a long-term pain-relieving effect. While thermal ablation may result in sustained pain relief, loss of pudendal motor function, main sensory sexual functions, and pain recurrence may occur (18). Thus, surgical decompression and neurectomy of the perineal sensory branches represented the best option. As demonstrated in this case, surgical decompression and sensory perineal branch neurectomy can provide longerterm pain relief and preservation of sexual function.

In conclusion, we report a case of pudendal nerve injury secondary to brachytherapy seeds placed outside the prostatic capsule. MRN visualized the extra-prostatic seeds and otherwise normal pudendal nerve. MRNguided nerve blocks proved valuable for this patient as they provided functional proof that the patient's pain was pudendal nerve-mediated, which was corroborated by histopathology demonstrating perineural fibrosis suggestive of inflammation and perineural scarring. As brachytherapy seeds are not retrievable, neurectomy of the sensory branches was curative resulting in long-lasting pain relief.

\section{Acknowledgments}

The authors would like to thank the patient for allowing them to share his case.

Funding: None.

\section{Footnote}

Reporting Checklist: The authors have completed the CARE checklist. Available at http://dx.doi.org/10.21037/ tau.2020.03.22

Conflicts of Interest: All authors have completed the ICMJE uniform disclosure form (available at http://dx.doi. org/10.21037/tau.2020.03.22). JF reports grants, personal fees and non-financial support from Siemens Healthcare USA, personal fees and non-financial support from General Electric Healthcare, non-financial support from Zimmer, non-financial support from DePuy, outside the submitted work. The other authors have no conflicts of interest to declare.

Ethical Statement: The authors are accountable for all aspects of the work in ensuring that questions related to the accuracy or integrity of any part of the work are appropriately investigated and resolved. The data presented in this case report was obtained under an internal review board-approved protocol for prospective data collection and with informed consent from the patient.

Open Access Statement: This is an Open Access article distributed in accordance with the Creative Commons Attribution-NonCommercial-NoDerivs 4.0 International License (CC BY-NC-ND 4.0), which permits the noncommercial replication and distribution of the article with the strict proviso that no changes or edits are made and the original work is properly cited (including links to both the formal publication through the relevant DOI and the license). See: https://creativecommons.org/licenses/by-nc-nd/4.0/.

\section{References}

1. Chen AB, D'Amico AV, Neville BA, et al. Patient and Treatment Factors Associated With Complications After 
Prostate Brachytherapy. J Clin Oncol 2006;24:5298-304.

2. Zaorsky NG, Davis BJ, Nguyen PL, et al. The evolution of brachytherapy for prostate cancer. Nat Rev Urol 2017;14:415-39.

3. Antolak SJ. Pudendal Neuralgia. In: Potts JM. editor. Genitourinary Pain and Inflammation: Diagnosis and Management. Totowa, NJ: Humana Press, 2008:39-56.

4. Antolak SJ. Re: Health Related Quality of Life in Men With Prostate Cancer. J Urol 2003;170:2391-2.

5. Antolak SJ, Hough DM, Pawlina W. The Chronic Pelvic Pain Syndrome After Brachytherapy for Carcinoma of the Prostate. J Urol 2002;167:2525.

6. Dellon AL, Coady D. Vulvar and pelvic pain terminology review: implications for microsurgeons. Microsurgery 2015;35:85-90.

7. Dellon AL. Pain with sitting related to injury of the posterior femoral cutaneous nerve. Microsurgery 2015;35:463-8.

8. Fritz J, Dellon AL, Williams EH, et al. 3-Tesla High-Field Magnetic Resonance Neurography for Guiding Nerve Blocks and Its Role in Pain Management. Magn Reson Imaging Clin N Am 2015;23:533-45.

9. Ahlawat S, Stern SE, Belzberg AJ, et al. High-resolution metal artifact reduction MR imaging of the lumbosacral plexus in patients with metallic implants. Skeletal Radiol 2017;46:897-908.

10. Barry MJ, Fowler FJ Jr, O'leary MP, et al. The American Urological Association Symptom Index for Benign

Cite this article as: Bonham LW, Herati AS, McCarthy EF, Dellon AL, Fritz J. Diagnostic and interventional magnetic resonance neurography diagnosis of brachytherapy seedmediated pudendal nerve injury: a case report. Transl Androl Urol 2020;9(3):1442-1447. doi:10.21037/tau.2020.03.22
Prostatic Hyperplasia. J Urol 2017;197:S189-97.

11. Fritz J, Chhabra A, Wang KC, et al. Magnetic Resonance Neurography-Guided Nerve Blocks for the Diagnosis and Treatment of Chronic Pelvic Pain Syndrome. Neuroimaging Clin N Am 2014;24:211-34.

12. Dellon AL, Wright EJ, Manson PN. Chronic pelvic pain after laser prostatectomy: treatment by resection of the perineal branches of the pudendal nerve. J Reconstr Microsurg 2014;30:547-50.

13. Labat JJ, Riant T, Robert R, et al. Diagnostic criteria for pudendal neuralgia by pudendal nerve entrapment (Nantes criteria). Neurourol Urodyn 2008;27:306-10.

14. Stav K, Dwyer PL, Roberts L. Pudendal neuralgia. Fact or fiction? Obstet Gynecol Surv 2009;64:190-9.

15. Robert R, Labat JJ, Bensignor M, et al. Decompression and Transposition of the Pudendal Nerve in Pudendal Neuralgia: A Randomized Controlled Trial and LongTerm Evaluation. Eur Urol 2005;47:403-8.

16. Prologo JD, Lin RC, Williams R, et al. Percutaneous CT-guided cryoablation for the treatment of refractory pudendal neuralgia. Skeletal Radiol 2015;44:709-14.

17. Bittman RW, Peters GL, Newsome JM, et al. Percutaneous Image-Guided Cryoneurolysis. AJR Am J Roentgenol 2018;210:454-65.

18. Yoon JHE, Grechushkin V, Chaudhry A, et al. Cryoneurolysis in Patients with Refractory Chronic Peripheral Neuropathic Pain. J Vasc Interv Radiol 2016;27:239-43. 


\section{Patient Care Timeline}

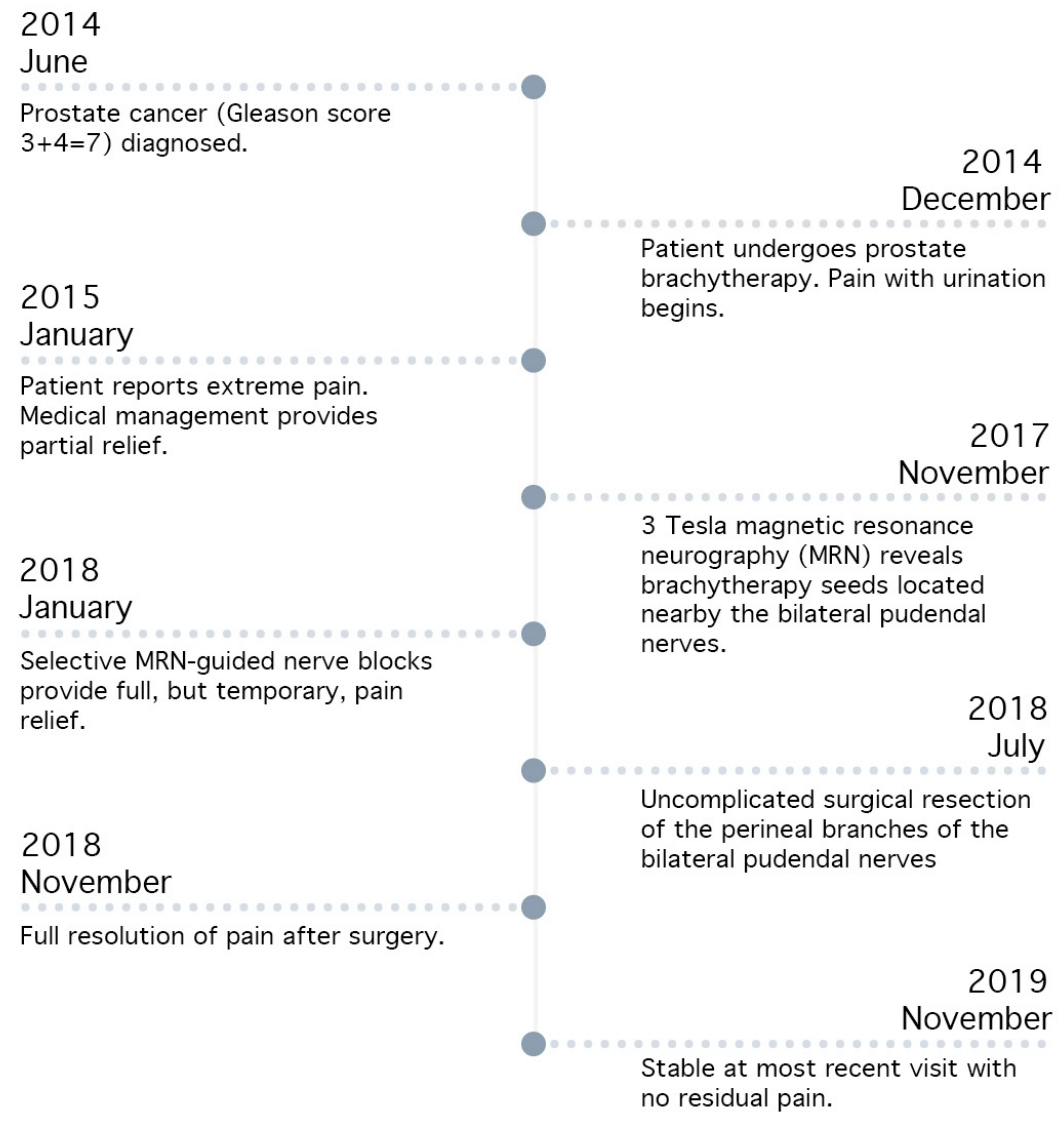

Figure S1 Patient care timeline illustrating the patient's clinical course from initial prostate cancer diagnosis to most recent visit. 